\title{
Strain-Release Arylations for the Bis-Functionalization of Azetidines
}

\author{
Florian Trauner, ${ }^{\text {a }}$ Felix Reiners, ${ }^{a^{\dagger}}$ Kodjo-Edmond Apaloo-Messan, ${ }^{b}$ Benedikt Nißl, ${ }^{a}$ Muhammad \\ Shahbaz, ${ }^{a}$ Jiang Dongfang, ${ }^{a}$ Julian Aicher, ${ }^{a}$ Dorian Didier ${ }^{a *}$
}

The addition of nucleophilic organometallic species onto in situ generated azabicyclobutanes enables the selective formation of 3 arylated azetidine intermediates through strain-release. Single pot strategies were further developed for the $\mathrm{N}$-arylation of resulting azetidines, employing either $\mathrm{S}_{\mathrm{N}} \mathrm{Ar}$ reactions or Buchwald-Hartwig couplings.

Strained $\mathrm{sp}^{3}$-rich heterocycles have emerged as important scaffolds in medicinal chemistry over recent years, as they can help tune pharmacokinetic properties.[1] Among those, azetidines have received particular attention. They have been used in numerous occasions as phenyl isosteres or to rigidify amine structures.[2]

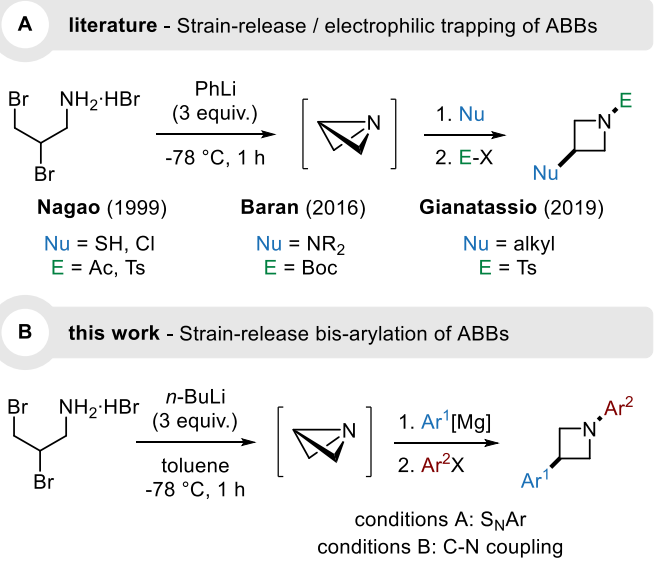

Scheme 1 Synthetic access to 1,3-bisfunctionalized azetidines.

Diverse strategies allow for the synthesis of substituted azetidines, including [2+2]-cycloadditions,[3] ring expansions,[4] ring contractions[5] and ring closures.[6] In addition, we recently contributed to this field through manipulation of unsaturated analogs $-2 \mathrm{H}$-azetines - via DielsAlder cycloadditions or hydrogenation reactions. [7]

Due to their ring strain, easily-accessible 1-azabicyclo[1.1.0]butanes (ABB) possess an interesting reactivity towards nucleophiles, representing therefore an interesting platform for further functionalizations. Given the high strain in $\mathrm{C}-\mathrm{N}-\mathrm{C}$ dihedral angles, $\mathrm{ABB}$ are strong nucleophiles and should readily react with electrophilic species. Following the pioneering work of Funke, [8] the group of Nagao exploited the concept of strain-release on $A B B$ with nucleophiles such as halogens and thiols, in the presence of $N$-trapping reagents like acyl- and tosyl chlorides (Scheme 1A).[9] In 2016, Baran developed an efficient strain-release amination protocol based on the use of primary and secondary turbo-amides as nucleophiles, for the late-stage diversification of drug-like molecules.[10] More recently, Gianatassio and co-workers exemplified the nucleophilic addition of Grignard reagents in the formation of the corresponding 3-alkylazetidines when using $\mathrm{Boc}_{2} \mathrm{O}, \mathrm{Ts} \mathrm{Cl}$ or acyl chlorides as electrophiles.[11] 1,2Boronate rearrangements also proved efficient for the substitution of azetidines at position 3 from ABB.[12]

Surprisingly, molecules as simple as 1,3-bisarylated azetidines are scarcely found in the literature, which certainly stems from the difficulty to access their structure efficiently and selectively. Despite efforts mentioned above, the introduction of aryl moieties at position 3 of $A B B$ through strain-release remains undisclosed. With a general interest in 4-membered carbo- and heterocycles and their implication in drug discovery,[13] we set out to establish a reliable method for the construction of such functionalized azetidine architectures.

Our investigations started with the examination of the ringopening reactions of $A B B$ with ex situ generated aryl-Grignard reagents. In previous reports, AAB were generated in THF by double cyclization, employing an excess of phenyl lithium as base for deprotonation. First experiments were performed following these conditions (PhLi, THF), revealing however the formation of several by-products. The expected product 3, obtained via nucleophilic addition of $p$-Tol $\mathrm{MgBr}$ was only produced in low yields and could not be isolated from $\mathbf{4}$ and $\mathbf{5}$, generated by the nucleophilic additions of residual PhLi and THF-soluble $\mathrm{LiBr}$, respectively.

\footnotetext{
a. Department of Chemistry and Pharmacy, Ludwig-Maximilians-Universität

München, Butenandtstraße 5-13, 81377 Munich, Germany.

b. Université Paris-Saclay, 91405 Orsay, France.

+ These authors contributed equally.
} 
A Issues associated with reported conditions

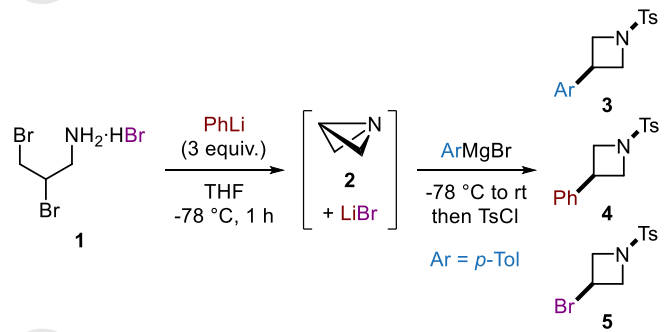

B New set of conditions
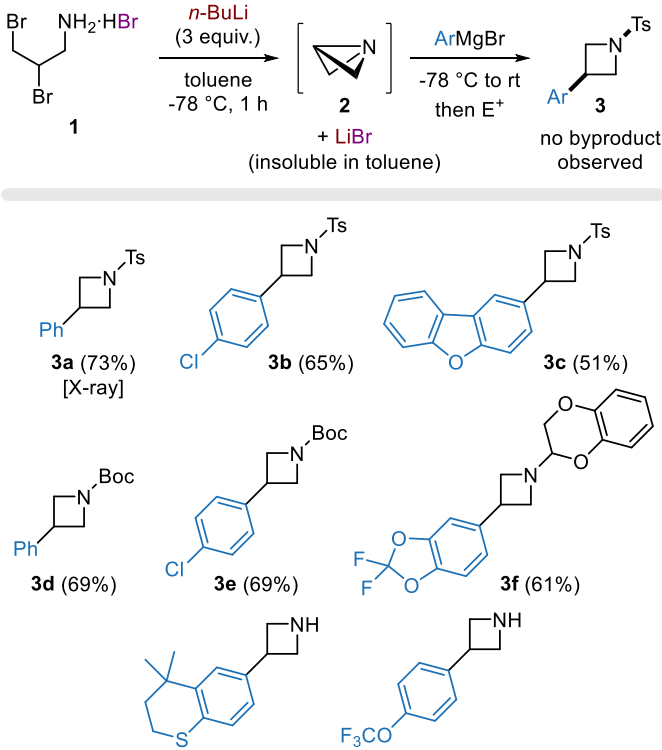

$3 g(62 \%)$ fluoropyridine to give $\mathbf{7 a}$ in $80 \%$ yield. Varying the substitution pattern of 2-fluoropyridines yielded halogenated compounds $\mathbf{7 b}$ and $\mathbf{7 c}$, nitrile derivative $\mathbf{7 d}$, and 2 -fluoroquinoline gave $\mathbf{7 e}$ in $70 \%$ yield. Electron-rich ( $p$-OMe, $p$-SR) and electron-poor ( $p$ F) aryl-Grignard reagents were also proven efficient in this transformation, furnishing $\mathbf{7 f - h}$ in 40 to $72 \%$ yield. The introduction of heteroaromatic was also exemplified with 2 thiophenylmagensium reagents, providing $7 \mathbf{i}$ (65\%).
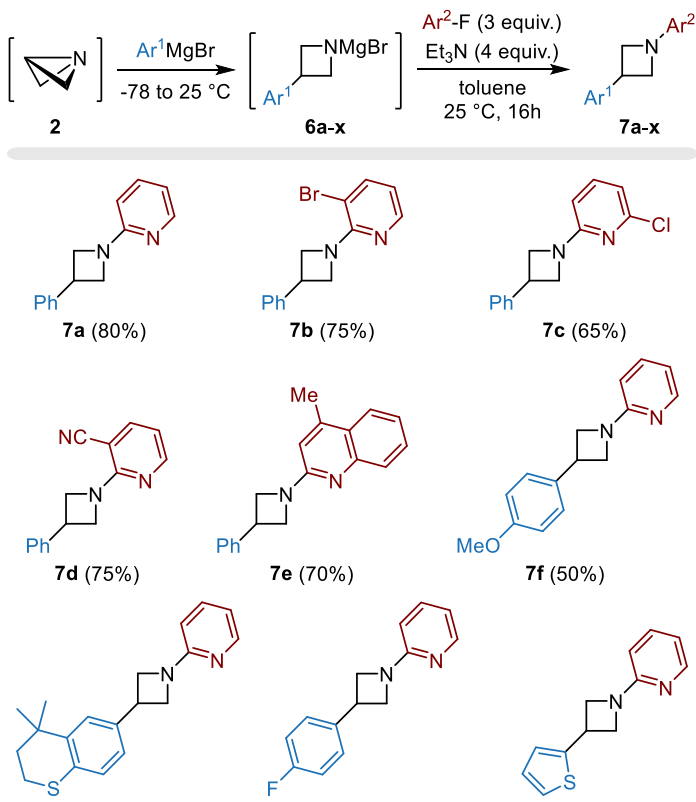

$7 g(40 \%)$

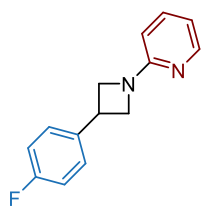

7h $(72 \%)$

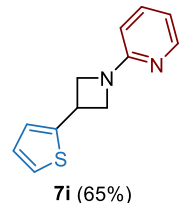

Scheme 3 3-Arylazetidines in nucleophilic aromatic substitutions $\left(S_{N} A r\right)$.

Scheme2 Establishment of conditions for the strain-release reaction with arylmagnesium reagents.

We envisioned that PhLi could be replaced by $n$-BuLi, avoiding therefore the formation of inseparable molecule 4, $n$-BuLi being more basic, yet less nucleophilic than PhLi. Preventing the formation of $\mathbf{5}$ was achieved by switching to toluene as solvent to precipitate $\mathrm{LiBr}$ formed during the cyclization process, therefore inhibiting its undesired reactivity with $\mathbf{2}$. With a new set of conditions in hands, products 3a-i were isolated without byproducts in 51-73\% yields, upon addition of electrophiles like $\mathrm{TsCl}$ and $\mathrm{Boc}_{2} \mathrm{O}$ or through simple hydrolysis. Importantly, the unprecedented nucleophilic addition of aryl-Grignard reagents onto ABB proved efficient, with a smooth introduction of aryl moieties at position 3 . We envisioned that the intermediate magnesium amide 6 resulting from a ring opening reaction could be used in situ in a further nucleophilic aromatic substitution onto electron-deficient aromatics, providing a first approach to bis-arylated structures. 2-Fluorinated pyridines were chosen as appropriate substrates for this transformation, given that their electron-deficient nature allow for the stabilization of the intermediate Meisenheimer complex. After successful generation of the $\mathrm{N}$-azetidinyl-magnesium species $\mathbf{6 a}$ in situ - with $\mathrm{PhMgBr}$ - further $\mathrm{S}_{N} A r$ was performed in the presence of triethylamine and an excess of the electrophilic 2-
Although this strategy proved efficient, it only allows for the substitution of fluorides on pyridine-like electrophiles. Other electron-deficient aryls possessing cyano, nitro or ester groups failed to generate the desired $\mathrm{N}$-arylated azetidines, consequently limiting the scope of the reaction. Therefore, we looked for an alternative that would broaden the possibilities for $\mathrm{C}-\mathrm{N}$ bond formation and oriented ourselves towards $\mathrm{Pd}$ catalyzed transformations. Buchwald-Hartwig coupling reactions have witnessed tremendous improvements since the pioneering studies of those who gave their names to the transformation.[14] Many examples from drug discovery programs have been reported on the coupling of secondary amines,[15] even including some azetidines.[12,16]

Two starting materials were tested for reaction optimizations (Table 1), azetidinium-oxalate salt (8), following Carreira's procedure on spiro compounds,[16] and free azetidine (9), isolated through basic workup, using $p$-bromoanisole as coupling partner. While catalytic systems such as $\mathrm{Pd}_{2}(\mathrm{dba})_{3} / \mathrm{BINAP}$ or $\mathrm{Pd}(\mathrm{OAc})_{2} /$ Xantphos (entries 1-3) only yielded coupling product $\mathbf{1 0}$ in 4 to $17 \%$ from the oxalate salt 8 (with either $\mathrm{KO}$-Bu or $\mathrm{NaOt}$-Bu as the base), it was isolated in 22 to $24 \%$ yield when switching to $x$ PhosPdG3/Brettphos and RuPhosPdG3/RuPhos (entries 4 and 5), respectively. Although full consumption of the coupling partner was observed in most cases, and despite further optimization efforts using $\mathbf{8}$, no 
better yield than $24 \%$ could be obtained, probably because of undesired ring-opening side-reactions. However, when employing the free azetidine 9 (entries 6-8), yields were increased to $82 \%$ in the presence of $x$ PhosPdG3/Brettphos as the catalytic system. If lower temperatures resulted in lower yields, decreasing the catalyst loading to $1 \%$ did not show any negative influence on the efficiency of the coupling.

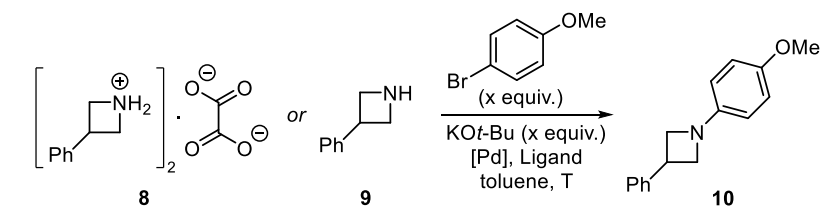

\begin{tabular}{|c|c|c|c|c|}
\hline Entry & Substrate & {$[\mathrm{Pd}] /$ ligand } & $\begin{array}{c}\mathrm{T} \\
\left({ }^{\circ} \mathrm{C}\right)\end{array}$ & $\begin{array}{c}\text { Yield } \\
(\%)\end{array}$ \\
\hline 1 & $\mathbf{8}$ & $\mathrm{Pd}_{2}(\mathrm{dba})_{3} / \mathrm{BINAP}^{(\mathrm{a})}$ & 100 & 17 \\
\hline 2 & $\mathbf{8}$ & $\mathrm{Pd}_{2}(\mathrm{dba})_{3} / \mathrm{BINAP}^{(\mathrm{a})}$ & 100 & $16^{(\mathrm{b})}$ \\
\hline 3 & $\mathbf{8}$ & $\mathrm{Pd}(\mathrm{OAc})_{2} /$ Xantphos $^{(\mathrm{a})}$ & 100 & 4 \\
\hline 4 & $\mathbf{8}$ & xPhosPdG3/Brettphos $^{(\mathrm{c})}$ & 100 & 24 \\
\hline 5 & $\mathbf{8}$ & RuPhosPdG3/RuPhos $^{(\mathrm{c})}$ & 100 & 22 \\
\hline 6 & $\mathbf{9}$ & xPhosPdG3/Brettphos $^{(\mathrm{c})}$ & 100 & 82 \\
\hline 7 & $\mathbf{9}$ & xPhosPdG3/Brettphos $^{(\mathrm{c})}$ & 80 & 73 \\
\hline 8 & $\mathbf{9}$ & RuPhosPdG3/RuPhos $^{(\mathrm{c})}$ & 100 & 71 \\
\hline
\end{tabular}

${ }^{a}$ Reactions performed with $3 \mathrm{~mol} \%[\mathrm{Pd}],[\mathrm{Pd}] /$ ligand $=1.5: 1,{ }^{b} \mathrm{NaOt}$-Bu instead of $\mathrm{KOt}$-Bu, ${ }^{\mathrm{C}}$ Reactionsperformed with $1 \mathrm{~mol} \%[\mathrm{Pd}],[\mathrm{Pd}] /$ ligand $=1: 1$.

Table 1 Optimizations on Buchwald-Hartwig couplings of azetidines.

With optimal conditions in hands, the scope of BuchwaldHartwig coupling on ex situ generated 3-arylazetidines was evaluated in the presence of diversely substituted aryl and heteroaryl bromides. 3-Phenylazetidines performed equally well with electron-rich and electron-poor aryl bromides, providing 1,3-bis-arylated azetidines 9a-g in excellent yields ( 82 to $99 \%)$. Worthy of note, a better yield was obtained through Bachwald-Hartwig coupling on 2-bromopyridines (9g, 99\%) than under $\mathrm{S}_{\mathrm{N}} \mathrm{Ar}$ conditions (7a-i, 40 to $80 \%$ ). The introduction of a donor group $\left(\mathrm{OCF}_{3}\right)$ at the para-position similarly led to compounds $\mathbf{9 h}-\mathbf{I}$ in moderate to good yields (up to $86 \%$ ). The reaction also proved highly functional group tolerant, as witnessed in the case of $\mathbf{9 k}$ containing an aldehyde (59\%), and selective for bromides over chlorides (9i, 77\%). Heterocyclic Grignard reagents such as 2-thiophenymagnesium and 3dibenzothiophenylmagnesium bromides were also engaged in bis-arylations (9m-0, 46 to $62 \%$ ). The addition of an electronpoor Grignard reagent provided $\mathbf{9 q}$ in $\mathbf{7 9 \%}$ yield. Analogs of drug compounds were finally synthesized following this rational design, to demonstrate the applicability of such method. Azetidine-analogs (9q-9t) of Adapalene and Tazarotene were obtained in a minimum of steps in very good yields through Buchwald-Hartwig couplings, in which the azetidine-moiety was employed to serve as isostere of either aryl- or alkynyl-groups.
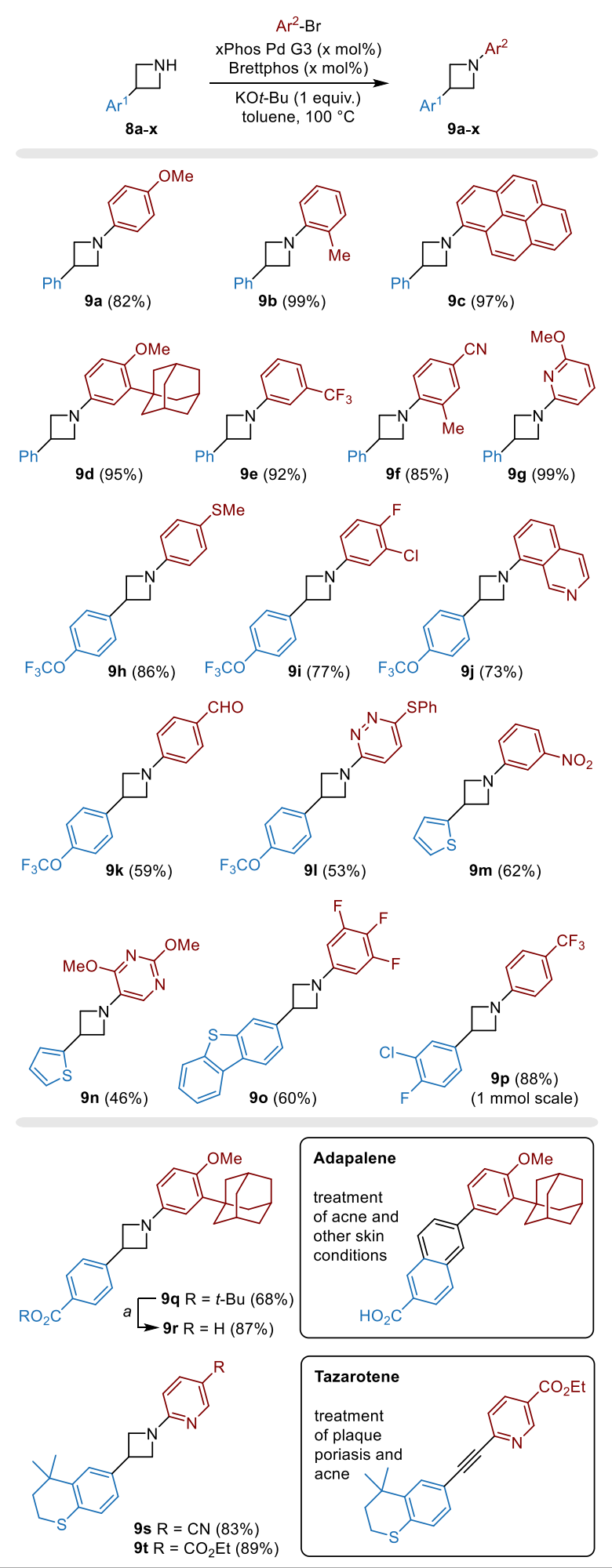

Scheme 4 Buchwald-Hartwig coupling of 3-arylazetidines.

As the process for azetidine bis-arylation through coupling strategies led to a library of widely functionalized molecules, we next aimed at increasing step-economy by withdrawing the intermediate purification of the free azetidine. A one-pot sequence was therefore developed to enable the bis-arylation of azetidines directly from 1, given that both steps of strain- 
release and Buchwald-Hartwig coupling are performed in the same solvent system. In situ generation of ABB $\mathbf{2}$ was followed by ring opening with aryl magnesium species, and finally engaged in the coupling reaction under Pd-catalysed conditions described above without purification (Scheme 5). Compounds 7a and 9a were isolated in slightly lower yields than in the twostep procedure. Varying the nature of aryl Grignard reagents led to functionalized scaffolds $\mathbf{1 0 a - f}$ in moderate to good yields after Buchwald-Hartwig C-N coupling with aryl bromides, including substituted phenyl, pyrazine and quinoline moieties.
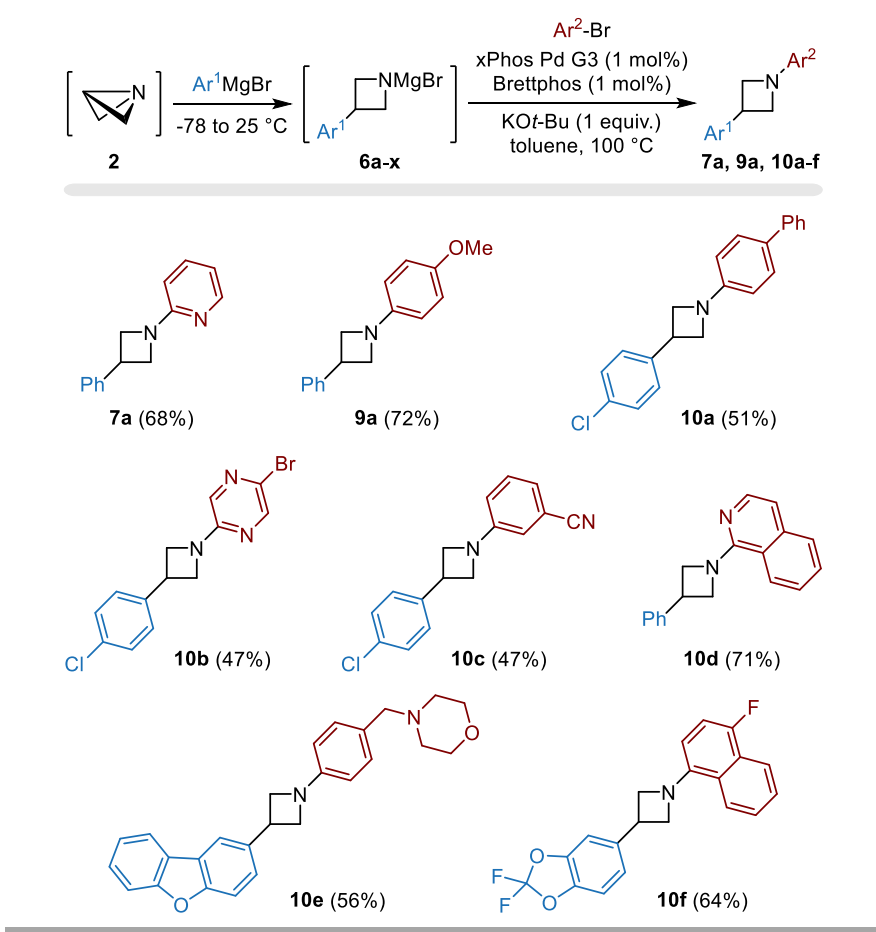

Scheme 5 One-pot ABB ring-opening / Buchwald-Hartwig coupling sequence.

In summary, we have developed a very efficient procedure for the 1,3-bis-arylation of azetidines, taking advantage of in situ generated reactive strained aza-bicyclobutanes. Unprecedented arylation at position 3 was performed with arylmagnesium reagents, adapting the solvent system to avoid undesired products, and demonstrating the high tolerance of the method for functional groups. Simple and versatile $\mathrm{C}-\mathrm{N}$ bond formation strategies were applied in a single-pot sequence, allowing the design of a vast library of building blocks, including analogs of drug compounds, taking a step further towards the challenging implementation of rigid $\mathrm{sp}^{3}$-rich scaffolds in drug-discovery programs.

\section{Acknowledgments}

D.D., F.T. and F.R. are grateful to the Fonds der Chemischen Industrie, the Deutsche Forschungsgemeinschaft (DFG grant: DI 2227/2-1 and Heisenberg fellowship: DI 2227/4-1) and to the Ludwig-Maximilians University (LMU Excellence) for PhD funding and financial support. M.S. and J.D. thank the Punjab Educational Endowment Fund (PEEF) and the Chinese Scholar- ship Council (CSC) for the generous attributions of PhD fellowships. K.A. is grateful to the ERASMUS+ program.

\section{Notes and references}

1 (a) D. Wang and S. Gao, Org. Chem. Front. 2014, 1, 556. (b) C. Lamberth, Bioorg. Med. Chem. 2009, 17, 4047. (c) E. M. Carreira and T. C. Fessard, Chem. Rev. 2014, 114, 8257. (d) A. K. Simlandy, M.-Y. Lyu and M. K. Brown ACS Catal. 2021, 11, 12815. (e) T. W. Reidl and L. L. Anderson, Asian J. Org. Chem. 2019, 8, 931.

2 (a) H. Mughal and M. Szostak, Org. Biomol. Chem. 2021, 19, 3274. (b) D. R. Parmar, J. Y. Soni, R. Guduru, R. H. Rayani, R. V. Kusurkar and A. G. Vala, Arch Pharm. 2021, 354, 210062. (c) C. Brotherton-Pleiss, P. Yue, Y. Zhu, K. Nakamura, W. Chen, W. Fu, C. Kubota, J. Chen, F. Alonso-Valenteen, S. Mikhael, L. Medina-Kauwe, M. A. Tius, F. Lopez-Tapia and J. Turkson, J. Med. Chem. 2021, 64, 695. (d) N. A. Meanwell, J. Med. Chem. 2011, 54, 2529. (e) A. Brown, D. Ellis, O. Wallace and M. Ralph Bioorg. Med. Chem. Lett. 2021, 20, 1851.

3 Selected examples: (a) J.-B. Denis, G. Masson, P. Retailleau and J. Zhu, Angew. Chem. Int. Ed. 2011, 50, 5356. (b) T. Nishimura, Y. Yasuhara and T. Hayashi, Angew. Chem. Int. Ed. 2006, 45, 5164.

4 Selected examples: (a) S. C. Schmid, I. A. Guzei and J. M. Schomaker, Angew. Chem. Int. Ed. 2017, 56, 12229. (b) J.-Q. Han, H.-H. Zhang, P.-F. Xu and Y.-C. Luo, Org. Lett. 2016, 18, 5212. (c) S. Malik and U. K. Nadir, Synlett 2008, 108.

5 N. Kern, A.-S. Felten, J.-M. Weibel, P. Pale and A. Blanc, Org. Lett. 2014, 16, 6104.

6 Selected examples: (a) P. Quinodoz, B. Drouillat, K. Wright, J. Marrot and F. Couty, J. Org. Chem. 2016, 81, 289. (b) R. M. de Figueiredo, R. Fröhlich and M. Christmann, J. Org. Chem. 2006, 71, 4147. (c) S. P. Fritz, J. F. Moya, M. G. Unthank, E. M. McGarrigle and V. K. Aggarwal, Synthesis 2012, 44, 1584

7 (a) A. Music, A. N. Baumann, M. Eisold and D. Didier, J. Org. Chem. 2018, 83, 783. (b) A. N. Baumann, F. Reiners, T. Juli and D. Didier, Org. Lett. 2018, 20, 6736. (c) F. Reiners, E. Joseph, B. Nißl and D. Didier, Org. Lett. 2020, 22, 8533.

8 W. Funke, Angew. Chem. Int. Ed. 1969, 8, 70.

9 K. Hayashi, C. Sato, S. Hiti, T. Kumagai, S. Tamai, T. Abe and Y. Nagao, Tetrahedron Lett. 1999, 40, 3761.

10 R. Gianatassio, J. M. Lopchuk, J. Wang, C.-M. Pan, L. R. Malins, L. Prieto, T. A. Brandt, M. R. Collins, G. M. Gallego, N. W. Sach, J. E. Spangler, H. Zhu, J. Zhu, P. S. Baran, Science 2016, 351, 241.

11 R. Gianatassio, D. Kadish, Org. Lett. 2019, 21, 2060.

12 A. Fawcett, A. Murtaza, C. H. U. Gregson and V. K. Aggarwal, J. Am. Chem. Soc. 2019, 141, 4573.

13 D. Didier and F. Reiners, Chem. Rec. 2021, 21, 1144

14 (a) J. Louie and J. Hartwig, Tetrahedron Lett. 1995, 36, 3609. (b) A. S. Guram, R. A. Rennels and S. L. Buchwald, Angew. Chem. Int. Ed. 1995, 34, 1348.

15 P. Ruiz-Castillo and S. L. Buchwald, Chem. Rev. 2016, 116, 12564.

16 J. A. Burkhard, B. Wagner, H. Fischer, F. Schuler, K. Müller and E. M. Carreira, Angew. Chem. Int. Ed. 2010, 49, 3524. 